\title{
Agradecimento aos pareceristas
}

A equipe da Revista da ABENO agradece aos seguintes colegas pela revisão de artigos em 2019:

Aline Macarevich Condessa

Aluísio Ferreira Celestino Júnior

Ana Carolina Oliveira Peres

Ana Cláudia Pereira dos Santos

Ana Cláudia Cimardi

André Ulisses Dantas Batista

André Cavalcante da Silva Barbosa

Andreas Rucks Varvaki Rados

Ângela Scarparo

Arlete Maria Gomes Oliveira

Armando Hayassy

Bianca Santiago

Camila Costa

Camila Helen Grock

Carla de Souza Oliveira

Carla Massignan

Caroline Solda

Caroline Stein

Eliane Medeiros Serpa

Elizandra Silva Penha

Eloá Rossoni

Fabiana Schneider Pires

Fabiane Alves Farias Guimarães

Fábio Barbosa de Souza

Fernando Bittencourt

Flávia Martão Flório

Gêisa Sampaio

Graciela Soares Fonseca

Grasiela Garret da Silva

Gersinei Carlos Freitas

Ismar Eduardo Martins-Filho

Janaína Rocha de Sousa Almeida
Joana Danielle Carneiro

Jocianelle Nunes

Karen Barea de Paula

Kevan Guilherme Nóbrega Barbosa

Leandro Brambilla Martorell

Lucas Pinheiro

Lucimar Aparecida Britto Codato

Luiz Carlos Machado Miguel

Márcia Cançado Figueiredo

Maria Cynésia Medeiros de Barros Torres

Maria Helena Chaves de Vasconcelos Catão

Maria Socorro Araújo Dias

Maria Eneide Almeida

Maria Goretti Queiroz

Marília Gerhardt-Oliveira

Mario Marques Fernandes

Mary Caroline Skelton-Macedo

Maurício Fernando Nunes Teixeira

Mirelle Finkler

Pablo Guilherme Caldarelli

Paulo Mauricio Reis de Melo Jr

Pedro Leme

Pedro Henrique Sette de Souza

Rafael Aiello Bomfim

Renata de Oliveira Cartaxo

Sergio Augusto Quevedo Miguens Jr

Sileno Brum

Talitha R. Ribeiro Fernandes Pessoa

Thaíse Araujo

Thaíse Gomes e Nóbrega 